\title{
REFLEXIONES EN TORNO A LA SOCIEDAD, VIOLENCIA Y EDUCACIÓN
}

\author{
Marisa VÁzquez Martínez \\ Alicia PÉrez Tarrés \\ Leonor M. Cantera Espinosa \\ Universitat Autònoma de Barcelona, Barcelona, España \\ Joilson Pereira da SiLVA \\ Universidade Federal de Sergipe (UFS), São Cristóvão, Sergipe, \\ Brasil
}

\begin{abstract}
RESUMEN: El presente artículo reflexiona sobre la necesidad imperiosa de cuestionar aquellos aspectos propios de una sociedad patriarcal como espacio de relación de los y las adolescentes. Fruto del trabajo de las investigaciones cualitativas que venimos desarrollando, se detecta la persistencia de un sistema patriarcal que dificulta el desarrollo de relaciones igualitarias. Se hace pues, necesario, conocer esta realidad para así plantear propuestas educativas que favorezcan el crecimiento de los niños y niñas en modelos de relación igualitarios.
\end{abstract}

Palabras Claves: Sociedad patriarcal. Violencia. Adolescencia. Educación.

\section{INTRODUCCIÓN}

Nos encontramos en un momento socio-cultural en el que la tendencia a la hora de abordar la comprensión de las problemáticas sociales es la de fragmentarla. Desde esta fragmentación parece que se hace más fácil 
la comprensión de los fenómenos; sin embargo, el comprender la realidad por parcelas, nos impide visibilizar realidades que están compactadas y que se van esparciendo como una mancha de aceite, calando en todos y cada uno de los sistemas que constituyen la sociedad: familia, escuela, trabajos, amistades, etc. Invisibilidad de la que nos previene la creadora del concepto interseccionalidad, Kimberle Williams Crenshaw, así como otras autoras y autores (BARBERA, 2016; EXPÓSITO MOLINA, 2012; SILVA SILVEIRA; CAETANO NARDI, 2014; SYMINGTON, 2004; WILLIAMS CRENSHAW, 1994).

La cultura patriarcal forma parte de un orden macro, mundial, que dificulta e invisibiliza una serie de comportamientos y actitudes entendidos como relaciones desiguales. La violencia, tal y como indica Cantera Espinosa (2004) "es camaleónica", adopta distintas formas, semblantes, de tal manera que se nos hace familiar e incluso natural y difícil de controlar el poder detectarla.

Observamos que, en el caso de los y las adolescentes, hablar de violencia requiere de una mirada amplia, socio-contextual. La violencia más investigada es aquella que se da: (a) en las relaciones de pareja, (b) hacia los progenitores (violencia ascendente) y/o (c) violencia entre iguales (bullying).

Para efecto de este escrito, analizaremos aspectos relacionados con la violencia a través de la población adolescente pues es, precisamente en ella, en quien se han centrado los esfuerzos y sobre quien han recaído las campañas de educación no violenta.Y, porque es a nuestros/as jóvenes a quienes exigimos y sobre quienes proyectamos la esperanza de una convivencia más justa. Sin detenernos, mostramos la violencia en la relación íntima de pareja juvenil, la violencia entre iguales (o bullying) y la violencia ascendente, como aquellaque se ejerce hacia los progenitores. Además, Ilamamos la atención de la violencia en contextos, paradójicamente, pensados para erradicarla.

Comenzando con el ámbito de la violencia en la relación de pareja en jóvenes, la Organización Mundial de la Salud (OMS, 2016) ya nos advierte que, de entre un 3 a un $24 \%$ de las mujeres, declaran que su primera experiencia sexual fue forzada. Fernández (2016) señala que, según la Consejería de Igualdad y el Instituto Andaluz de la Mujer, la violencia de género en adolescentes creció y como consecuencia se atendió a un 37,2\% más de mujeres adolescentes que el año anterior. Además, en el informe elaborado por Ruiz Repullo (2016) se muestra que los primeros signos de violencia de género en adolescentes se justifican en la idea de un amor patriarcal, lo cual ya nos indica una de las posibles razones de esta chocante realidad. En una revisión sistemática sobre la violencia de género en parejas jóvenes realizada por Joly y Connolly (2016), se expuso que el sentimiento de vulnerabilidad, 
la falta de respeto y los juicios de valor, así como las desigualdades en la relación fueron motivaciones para perpetrar la violencia en la pareja por parte de las jóvenes.

En relación con la violencia entre iguales o bullying, podemos comprobar que cada día son más las noticias en los medios de información que alertan sobre esta cuestión. En los años 90, Dan Olweus (OLWEUS, 2004) ya describe un tipo de violencia particular que se da en las escuelas, entre los mismos compañeros/as. Se caracteriza por un comportamiento agresivo (golpes, insultos, amenazas, difusión de rumores, etc.) emitido por parte de un/a agresor/a o un grupo. Fue necesario ponerle nombre a este tipo de comportamientos y analizar sus características y consecuencias, ya que hasta entoncesse consideraban normales y "cosas de jóvenes".

Save the Children, en el año 2016, llevó a cabo un macro estudio sobre Bullying y Ciberbullying en España (CALMAESTRA et al., 2016). Fueron encuestados 21.487 estudiantes de entre 12 y 16 años. Se puso de manifiesto que entre un $3 \%$ y un $10 \%$ del alumnado encuestado había sufrido algún tipo de maltrato por parte de sus iguales en la escuela mediante amenazas, insultos a través del móvil o acoso. Y lo que es más alarmante, la mitad de los/as encuestados/as reconoce haber insultado $u$ ofendido a un igual y uno de cada 3 reconoce haber agredido a algún/a compañero/a. En el estudio, se detectan también cuestiones de género, ya que las chicas aparecen como diana del bullying con más frecuencia que los chicos.

Cuando son preguntados por las razones que les llevan a ejercer estos comportamientos, sus respuestas son vagas, ya que expresan que no sabían que este comportamiento era violento, o bien que querían gastar una broma. Las víctimas expresan tres razones básicamente del por qué han sido objetos de bullying: para ser molestadas, por su aspecto físico o porque les tienen "manía".

Distintos autores/as como Collell y Escudé (2007), Díaz-Aguado (2005), Garaigordobil, Martinez-Valderrey y Aliri (2013), Rigby (2000), Guo (2013), Pereira da Silva y Santana Barreto (2012) entre otros/as, coinciden en que el bullying supone una afectación importante en el desarrollo del/ la adolescente ya que genera aislamiento o exclusión social, absentismo escolar, descenso en el rendimiento académico, vivir bajo el miedo e incluso comportamientos suicidas. Todo ello requiere de estrategias eficaces de prevención, detección e intervención hacia una convivencia escolar libre de violencias.

Por último, pero no menos importante, se detecta un aumento en la violencia ascendente, es decir, aquella que ejercen hijos e hijas hacia sus progenitores. 
Creemos necesario, en primer lugar, delimitar correctamente el término violencia ascendente o violencia filio-parental (VFP), siendo aquella que se caracteriza por "conductas reiteradas de violencia física, psicológica (verbal o no verbal) o económica, dirigida a las y los progenitores, o a aquellas personas que ocupen su lugar (PEREIRA et al. , 2017, p.8). Quedan excluidos aquellos comportamientos ejecutados por jóvenes con algún tipo de patología severa, adicción o trastorno. Siguiendo a Cotterll (2001, citado en ORTEGA, 2015), estos actos tienen como objetivo causar daño físico y/o emocional, y financiero generando temor en los padres y permitiendo a los/ as agresores/as una posición de dominio y control

Este modo de ejercer la violencia convergeen un punto común propio de cualquier expresión de la violencia: ejercer el poder y el control, en este caso hacia las personas adultas de la familia, especialmente los progenitores $y$, con mayor frecuencia hacia las madres, abuelas o figuras femeninas de cuidado (MARTínEZ et al., 2015). Distintos estudios evidencian la frecuencia de maltratador varón frente a las mujeres, sin embargo otros, no establecen la diferencia en la cuestión de género sino en el modo en cómo se ejerce la violencia: la violencia ejercida por los varones es predominantemente física y verbal, mientras que en las mujeres es de tipo psicológico y financiero (AROCA, 2013; GONZÁLEZ-ÁLVAREZ et al., 2010).

Nos preguntamos, pues: ¿Qué está sucediendo para que después de años de implementación de políticas de prevención y concienciación de la violencia, sucedan estos hechos? ¿Dónde nos encontramos para que la violencia en las relaciones juveniles vaya en aumento? ¿Son conscientes los y las profesionales que trabajan en este ámbito que la violencia nos abarca a todos y todas, que nos posicionamos ante ella de tal manera que quizás no somos capaces de detectarla?

Responder a todas las preguntas previas sobrepasa los márgenes de este escrito. No obstante, deseamos hacer una aproximación a ellas focalizándonos en aspectos relacionado con estas violencias, mostrando lo arrojado en dos de las investigaciones que se han desarrollado en el grupo de investigación sobre la violencia en relaciones afectivas de pareja y en el trabajo (VIPAT). Por un lado, las dificultades que los y las jóvenes tienen para desarrollar comportamientos igualitarios en sus relaciones de pareja lo que les sitúa en una zona de riesgo en la que se hacen más vulnerables a sufrir la violencia; y por otro, el caso de los y las profesionales que trabajan en la intervención para erradicar la violencia machista, en la que se reproducen, en sus equipos de trabajo, los comportamientos que pretenden erradicar. 
MARCO TEÓRICO

La violencia puede ser definida como una acción o inacción intencional y dañina (SANMARTíN, 2007) que puede ser tanto física como simbólica y que tiene un carácter hostil (CANTERA, 2004). Hoy por hoy, "la nueva definición de la violencia incluye también la aplicación indirecta (...) de una fuerza estructural que se traduce en explotación, imposición y dominio" (NAVARRO, 2013, p. 99).

Partiendo de estas definiciones, se asume que la violencia puede darse en diversos ámbitos (familiar, laboral, escolar, social, etc.) pero que está encuadrada dentro de un contexto más amplio, el macro-contexto (o sistema patriarcal) el cual favorece un binarismo género-sexo, legitimando las formas de producir o recibir violencia. Cantera, Pallarés y Selva (2013) señalan el contexto donde se ubica la violencia en el mundo actual, asumiendo que esta se trata de una herramienta de control permitida y legitimada; que asegura a quien la ejerce la perpetuación de su posición superior; $y$, por último, que refuerza la sensación de control, estatus, poder y dominio.

Entre las múltiples formas que puede tomar la violencia entendida como una herramienta, queremos poner atención a la violencia simbólica, pues la misma se define como una acción que se ejerce contra el individuo con su propia complicidad, incluso de forma inconsciente (BORDIEU; PASSERON, 1996), pues este poder simbólico se impone como un arbitrario cultural, creando dominantes y dominados (PEÑA COLLAZOS, 2009).

Esta violencia se da dentro de un sistema patriarcal que según Cantera $(1999,2005)$ como cualquier otro tipo de sistema sociocultural, determina las reglas del juego de la violencia concebible, permisible y exigible. El patriarcado se define como un modelo de estructura social donde predomina una estructura vertical, jerárquica, basada en la autoridad, la disciplina y la represión (CANTERA; PALLARES; SELVA, 2013) y donde los roles sexuales tienen una fuerte influencia en la construcción de la identidad personal y colectiva. La configuración de los deseos, necesidades, incluso la subjetividad, aspectos muy íntimos del individuo, están, también, atravesados por este orden androcéntrico y patriarcal (CUBELLS; CALSAMIGLIA, 2015).

\section{Metodología}

A continuación, se presentan dos investigaciones enmarcadas dentro del programa doctoral "Persona y Sociedad en el Mundo Contemporáneo" de la Universidad Autónoma de Barcelona a partir de las cuales emergieron reflexiones y puntos de confluencia en relación con la investigación, 
intervención y características de la violencia en diversos contextos. Es, precisamente a través de estos trabajos, que surge la necesidad de cuestionar los planteamientos actuales en torno al fenómeno de la violencia.

La primera de estas investigaciones se titula"Violencia, trabajoysalud" y, en ella, se trata de abordar cómo los equipos que están especializados en intervenir en casos de violencia machista reproducen la violencia contra la que están luchando.

La segunda titulada "El imaginario de igualdad en las relaciones de pareja juveniles", trata sobre el modo en cómo los y las jóvenes entienden la igualdad en las relaciones de pareja, de tal manera que puedan emerger mitos, prejuicios, creencias que ayuden a entender por qué persiste la violencia en las relaciones de parejajóvenes. Ambas investigaciones abogaron por el marco de la investigación cualitativa, pues esta promueve las acciones de observación e interpretación, a través del método inductivo para descubrir nuevos conceptos desde una perspectiva holística (QUINTANA, 2006).

Las características dela investigación cualitativa, radicada en la manera de diseñar e implementar los estudios, la forma de comprender y abordar las problemáticas sociales y su visión holística decantaron la elección de este tipo de metodología frente a otras opciones epistemológicas (CAMPOY; GOMES, 2015). Según Gimeno y Pérez (2009) se utiliza el método inductivo sin pretender probar teorías o hipótesis, sino generar nuevos enfoques o planteamientos.

En ambas investigaciones se ha utilizado la entrevista como técnica que permite conocer y evidenciar la comprensión de fenómenos sociales. Así como visibilizar aquellos comportamientos y actitudes que sustentan la violencia, el modo en cómo se ejercen relaciones desde el dominio y el control, tanto en el ámbito laboral como en el afectivo emocional (RUíz OLABUÉNAGA, 2012).

Los datos obtenidos en estas investigaciones han sido interpretados con perspectiva de género a través de la teoría fundamentada. Esta se describe como " la teoría derivada de datos recopilados de forma sistemática y analizados por medio de un proceso de investigación" (STRAUSS; CORBIN, 2002, p.21). En las dos investigaciones se tuvieron en cuenta los aspectos éticos relativos a la investigación; siguiendo las directrices que marca el Código de buenas prácticas en la Investigación de la Universitat Autònoma de Barcelona (2013). 


\section{ANÁLISIS Y REFLEXIONES DESDE DOS INVESTIGACIONES}

En la investigación "El imaginario de igualdad en las relaciones de pareja juveniles", recurrimos a 56 jóvenes, 20 chicos y 36 chicas, de edades comprendidas entre los 20 y 25 años. Todos/as, universitarios/as de la ciudad de Barcelona. A efectos de este artículo tomaremos los datos obtenidos en la entrevista grupal en la que se discutió en torno a cómo ellos y ellas entienden la igualdad y la desigualdad en las relaciones de pareja jóvenes.

Para llevar a cabo la gestión y análisis de los datos obtenidos, utilizamos el gestor de datos Atlas.ti 8. Para ello seguimos las orientaciones de Cantera y Alvarado (2010) así como a Corbin y Strauss (1994), de tal modo que se hace posible establecer relaciones significativas entre las mismas.

Tras el análisis de las entrevistas grupales, establecimos dos grandes familias temáticas: la igualdad y la desigualdad. Estas familias albergan, cada una, dos dimensiones: (a) Elementos constituyentes de la igualdad/ desigualdad. (b) Presentación de la igualdad/desigualdad en las relaciones de pareja. Dentro de cada dimensión se han identificado distintas categorías y subcategorías. Para efectos de este artículo presentamos la categoría Respetar, ubicada dentro de la dimensión de los elementos constituyentes de la igualdad.

Dentro de la categoría Respetarlos y las participantes incluían las subcategorías: aceptar, renunciar, tolerar y ceder, acciones en las que se evidenció un terreno lleno de ambigüedades que podían conducir a relaciones de dependencia, sumisión y control. A continuación, desarrollaremos la subcategoría ceder, ya que permitió visibilizar elementos sujetos a mitos y creencias facilitadores de relaciones desigualitarias en las que la violencia psicológica se asienta de manera invisible.

Los y las participantes de la investigación estuvieron de acuerdo en que el hecho de ceder tenía que ver con la aceptación de los intereses y gustos de la pareja y que así se favorecía la igualdad en la relación. Por ejemplo, PV4-G1 ${ }^{1}$ dice: "Lo que quería decir es que cuando hablas de respeto, de aceptar, es cómo aceptar mutuamente. O sea, tú estás diciendo que, si él tiene una actividad cada domingo y tú la aceptas, digamos que, dentro del aceptar, está también el ceder". Hasta aquí parecería que intentan aplicar la expresión coloquial de "hoy por ti y mañana por mí", tratando de buscar y encontrar ese equilibrio ideal en el respeto por los intereses, tiempos de ocio, aficiones del otro/a (HERNANDO, 2007; RUIZ REPULLO, 2016; BOSCH; FERRER, 2013; DÍAZ-AGUADO, 2012). 
Sin embargo, profundizando en la conversación, se pudo ver que el hecho de ceder suponía renunciar a uno/a mismo/a, con el objetivo de que el otro/a fuera feliz: chica P.M4- G1 nos dice:"Para mi ceder es tener que renunciar a algo que a ti te gusta, para poder mejorar la situación de tu pareja". Se evidencia la creencia de que, en la relación de pareja, con frecuencia, se deben anteponer las necesidades e intereses personales, por la felicidad de la pareja (BOSCH; FERRER;NAVARRO,2010).

Según esto, la pareja aparece como generosa y a la vez omnipotente ya que, con su renuncia, consigue la mejora del otro. Sin embargo, esta creencia le asigna a lamujer, la responsabilidad del bienestar del otro, aspecto este propio de una comprensión patriarcal según la cual la mujer tiene, o debe tener, la capacidad de aportar armonía, bienestar a la relación, e incluso desde el amor romántico, cambiar a su pareja. Este tipo de apreciaciones entrarían dentro de lo que son los mitos del amor romántico, mitos que favorecen la desigualdad y que generan un agotamiento psicológico importante en las mujeres que lo comparten (RODRÍGUEZ et al., 2013; CARO BLANCO, 2008; BOSCH; FERRER; NAVARRO, 2010).

El conjunto de estas creencias, de que la mujer debe renunciar a sus deseos por el bien del otro, responden a lo llamado por Lagarde $(\mathrm{BOSCH}$; FERRER, 2013) "mandato de género", según los cuales a las mujeres les corresponde el entendimiento emocional de la relación, considerándose que son ellas las que deben velar por la felicidad de la pareja, además de otras asignaciones, y que, según de Miguel (2008), responden a un sistema de creencias patriarcales generadoras de desigualdades en la relación de pareja. Una de las participantes también expone: “Por ejemplo, tú no te das cuenta de que siempre eres tú la que siempre está cediendo. Hasta que algún compañero tuyo te dice que abras los ojos, porque siempre eres tú la que tiene que, por ejemplo, cancelar tus planes para acoplarte a los de él, pero que é nunca lo hace por ti. O sea, que siempre tienes que acabar hacienda lo que él quiere" (P. M1 G1).

Podríamos afirmar que, en palabras de Bonino (2004), este pensar puede conducir a que estos/as jóvenes se vean involucrados/as en una violencia de baja intensidad que no se ve, pero que destruye; especialmente cuando este tipo de comportamientos se da en el hombre como una manera de mantener el control emocional de su pareja. Consecuentemente, si dentro de lo que se considera relaciones de pareja igualitarias, se entiende que respetar es aceptar, ceder, tolerar y renunciar por y para la felicidad de la otra parte, nos encontramos en una zona de riesgo para que el abuso se instaure y la deseada balanza del equilibrio se decante hacia un lado solamente. 
Si vamos más allá de la víctima y de quien ejecuta la violencia y llevamos la mirada a los espacios terapéuticos o de recuperación de la violencia machista, veremos que no existen lugares inmunizados contra la transmisión de la violencia, al tiempo que nos da algunas claves que conviene no perder de vista para su erradicación.

Cantera, (2002) nos advierte de la reproducción de la violencia en los senos de los centros donde se trabaja contra la misma; al ser lugares de micro reflejo social. Quiñones, Cantera y Ojeda (2013) indagaron en los significados que se construyen sobre las formas y estrategias que adquiere la violencia en espacios de trabajos donde se intentaerradicar, así como los contextos en los que no resulta sencillo desvelar o identificar la violencia. Los resultados de este estudio afirman que las participantes vivenciaron violencia principalmente psicológica, la cual produjo efectos negativos sobre la autoestima, la identidad personal y somatizaciones (p.ej. dolores de cabeza y mareos).

Por otro lado, en la investigación "Violencia, trabajo y salud" se entrevistaron a 20 profesionales que trabajan en centros en los que se pretende erradicar la violencia machista. Para efectos de este escrito, se van a presentar los resultados obtenidos en la categoría violencia en el trabajo, pues esta nos permite identificar las relaciones y dinámicas violentas que pueden establecerse en estos ambientes de forma, a veces, sutil.

Los elementos de análisis encontrados en la categoría violencia en el trabajo fueron: insultar, ignorar, cuestionar, no reconocer, chantajear, controlar, amenazar, tergiversar y malmeter. Esto significa, que las personas que participaron en el estudio describen que han sido víctimas o testigos de estas acciones en sus lugares de trabajo. Además, es importante destacar las formas en que para las personas entrevistadas adoptó esta violencia, pues lo relatan como una realidad muy cambiante y dependiente de las circunstancias, aspecto importante entre las particularidades de este fenómeno. Los siguientes fragmentos muestran situaciones maltratantes a través de las cuales operaban los/las compañeros/as del equipo: "Eran personas muy autoritarias, si no les dabas la razón, te hacían el vacío" (Cristina, p.1), "Esa actitud en la que todo el mundo opina sobre lo que se ha de hacer o se debería haber hecho... desde la imposición, desde el púlpito de la sabiduría..."(Carla, p. 5) o "Es tu cumpleaños, sé que lo es, y no tefelicito" (Helena, p.7).

En este caso, Cristina y Carla, describen el aislamiento de la persona como estrategia del agresor/a para imponer un relato o la versión de una situación, negando la diversidad de opiniones y la capacidad de la persona para decir o pensar libremente. O Helena, que nos cuenta cómo puede llegar 
el menosprecio y rechazo a través de una acción sutil e imperceptible para el resto del equipo. Si se puede reconocer y destacar un denominador común en todo ello es la intención de dañar al otro a través de diferentes mecanismos los cuales, a veces, son difíciles de definir, pero se sienten y sufren como algo injusto, externo, como algo que no se relaciona ni conjuga con ninguno de los actos que las víctimas pudieron realizar (QUIÑONES; CANTERA; OJEDA, 2013).

Otra de las características que los y las participantes resaltan está relacionada con el emisor y el receptor de las agresiones en sus lugares de trabajo. Nos parece interesante abordar esta cuestión pues está relacionada con la conciencia que cada uno de estos trabajadores/as tiene sobre su capacidad de generar y/o recibir acciones violentas.

La mayoría de ellas/os se reconocen en algún momento como víctimas, peromuy pocos reconocen ser capaces de maltratar o ser violentos/ as. Por ejemplo, Carla nos explica: "He sentido miedo a abusar de mi poder (...) en un momento dado yo me puedo poner en una posición de superioridad" (p.10) o Lidia: "Es muy difícil en estos aspectos el reconocer al otro, de defenderse, es perverso, pero cuando estás adentro...es muy, muy complejo..." (p.8). De estos relatos se desprende una reflexión sobre el potencial de la persona a ejercer violencia y su relación con el poder. También, la dificultad de ejecutar una respuesta no violenta ante un ataque, es decir, tener la capacidad y las herramientas para generar respuestas pacificas ante actitudes 0 acciones violentas.

Asimismo, para esta investigación, preguntamos por los motivos o explicaciones que los participantes atribuían al hecho de recibir o producir violencia en sus puestos de trabajo. Tras el análisis de su discurso podemos afirmar que entre los factores que más influyen en este fenómeno encontramos el aprendizaje personal de la violencia, desde la infancia, como una herramienta para alcanzar un objetivo dentro en el contexto sociocultural en el que estamos inmersos.

\section{ConClusión y BALANCE Final}

A comienzos de este artículo nos hacíamos las siguientes preguntas: ¿Qué está sucediendo para que después de años de implementación de políticas de prevención y concienciación de la violencia, sucedan estos hechos? ¿Dónde nos encontramos para que la violencia en las relaciones juveniles vaya en aumento? ¿Son conscientes los y las profesionales que trabajan en este ámbito que la violencia nos abarca a todos y todas, que nos posicionamos ante ella de tal manera que quizás no somos capaces de 
detectarla? Sin la pretensión de darle respuestas a todas y cada una de ellas; reflexionamos a partir de dos investigaciones.

La violencia sigue siendo, en palabras de Cantera (2004), camaleónica. Seguimos, como sociedad, siendo cómplices del sostén y reproducción de la misma. Nadie está inmune a ella, prueba de ello es el constatar que en centros donde se trabaja para erradicarla, en ocasiones tienelugar. Conceptos como igualdad, son vividos como avance, a la vez que son negación de la desigualdad y por lo tanto se convierten en mitos invisibilizadores.

Los datos que arrojan nuestras investigaciones, así como las investigaciones en materia de género, corroboran lo que autoras como Simón (2009), Santa Cruz (1992), Bodelón (2002), Facio (2009), Lagarde (2001), entre otras señalan sobre la estructura relacional patriarcal y hegemónica. Lo cultural impregna el modo en cómo nos relacionamos sea en el contexto que sea. Nuestras investigaciones confirman que a pesar de todo lo que se ha hecho hasta ahora en relación con la erradicación de la violencia todavía quedan cuestiones subterráneas, ocultas bajo la creencia de una igualdad conseguida. En palabras de Pujal y Garcia-Dauder"Por todo ello, consideramos que es necesario atender a estas transformaciones y renovadas formas de desigualdad y dominación, todavía muy invisibilizadas, e intentar comprender cómo están operando (PUJAL LLOMBART; GARCÍA-DAUDER, 2010. p.3).

Creemos que las preguntas iniciales requieren de una problematización, reflexión y acciones desde la perspectiva de género. A modo de conclusión presentamos, fruto de nuestras investigaciones, algunas pinceladas a considerar:

- Formar de manera rigurosa a profesionales de la educación, intervención y recuperación de la violencia bajo una perspectiva de género transversal a todos los espacios relacionales.

- Mantener el auto reciclaje formativo, al reconocer la habilidad transformadora que tiene la violencia.

- Vigilar la implementación de políticas transversales, tal y como se propuso desde el gender mainstreaming, que permitan abordar todos $y$ cada uno de los contextos de socialización.

- Situar y visibilizar la problemática de la violencia a través del marco cultural en el que estamos inmersos/as, sin fragmentarla ni aislarla del contexto social.

- Aumentar los espacios facilitadores de la toma de conciencia en torno a la necesidad de cuestionar el papel de cada una de las estructuras sociales en la reproducción de la violencia como pueden ser desde la 
escuela, la publicidad, los medios de comunicación, las redes sociales y los productos de ocio en general (cine, tv, salas de fiesta...), entre otros espacios educativos.

- Trabajar yreforzar los aprendizajes igualitarios, no sexistas ni estereotipados con todos los actores y actrices implicados/as en la socialización de la infancia y de jóvenes, por ejemplo: maestros/as, educadores/as, padres y madres,monitores/as, etc., pues se constata que estas creencias se sitúan en la base de la reproducción de la violencia juvenil. En esta misma línea, tener en cuenta aspectos sexistas y discriminadores hacia la mujer en el currículum escolar y modificarlos.

- Incluir en la escuela y en la orientación familiar estrategias de afrontamiento efectivas ante cualquier comportamiento que suponga, abuso y/o agresión.

\section{REFLEXÕES SOBRE SOCIEDADE, VIOLÊNCIA E EDUCAÇÃO}

RESUMEN: Este artigo reflete sobre a necessidade imperativa de questionar os aspectos de uma sociedade patriarcal como espaço de relação entre os adolescentes. Como resultado do trabalho de pesquisa qualitativa que estamos desenvolvendo, detecta-se a persistência de um sistema patriarcal que dificulta o desenvolvimento de relações igualitárias. Portanto, é necessário conhecer essa realidade para propor propostas educativas que favoreçam o crescimento de crianças em modelos de igualdade de relacionamento.

Palavras-chave: Sociedade patriarcal. Violência, Adolescência. Educação.

\section{REFLECTIONS AROUND SOCIETY, VIOLENCE AND EDUCATION}

ABSTRACT: This article reflects about the imperative need to question aspects of a patriarchal society as space of relationship among adolescents. Result of the work of qualitive research that we have been developing, there is detected the persistence of a patriarchal system that hinders the development egalitarian relationships. Therefore, it is necessary to know this reality in order to raise educational proposals that favor the growth of children in equal relationship models.

KEYWORDS: Patriarchal society. Violence. Adolescence. Education 
NotAs

1 Identificaremos al participante varón como PV y a la participante mujer como PM. Los datos de la siguiente investigación están bajo nombres ficticios.

\section{Referencias}

AROCA, C. La violencia de hijos adolescentes contra sus progenitores. Revista sobre la infancia y la adolescencia, n. 5, 2013, p. 12-30.

BARBERA, M. C. LA. Interseccionalidad, un "concepto viajero": orígenes, desarrollo e implementación en la Unión Europea. Interdisciplina, v. 4, n. 8, 2016, p. 105-122.

BODELÓN, E. Dos metáforas para la libertad: igualdad y diferencia. Anales de la Catédra Francisco Suárez, v. 36, 2002, p. 237-263.

BONINO, L. Micromachismos: La violencia Invisible en la pareja. La Cibeles. Ayuntamiento de Madrid, 2004, p. 19.

BORDIEU, P.; PASSERON, J. La reproducción; elementos para una teoría del sistema de enseñanza. Madrid, 1996.

$\mathrm{BOSCH}$, E.; FERRER, V. Del amor romántico a la violencia de género. Para una coeducación emocional en la agenda educativa. Profesorado. Revista de currículum y formación del profesorado, v. 17, 2013.

$\mathrm{BOSCH}$, E.; FERRER, V. Nuevo modelo explicativo para la violencia contra las mujeres en la pareja: el modelo piramidal y el proceso de filtraje. Asparkía, v. 24, n. Avances y retos actuales para combatir la violencia de género, 2013, p. 54-67.

BOSCH, E.; FERRER, V.; NAVARRO, C. Los mitos románticos en España. Boletín de psicología, n. 99, 2010, p. 7-31.

CALMAESTRA, J. et al.Yo a eso no juego. Bullying y ciberbullying en la infancia. España: Save the children, 2016.

CAMPOY, T. J.; GOMES, E. Técnicas e instrumentos cualitativos de recogida de datos. In: PANTOJA VALLEJO, A. (Ed.). Manual básico para la realización de tesinas, tesis y trabajos de investigación. $2^{\text {a }}$ edición ed. Madrid-España: Editorial EOS, 2015. p. 273-300.

CANTERA, L. M. Te Pego Porque Te Quiero. La violencia en la pareja. Universita ed. 1999.

CANTERA, L. M. La relación asistencial como interacción social. In: LÓPEZ BELTRÁN, M. T.; JIMÉNEZTOMÉ, M. J.; M ${ }^{\mathrm{a}}$, G. B. E. (Eds.). Violencia Géneronero. primera ed ed. Málaga: CEDMA, 2002. p. 391-397.

CANTERA, L.M. Más allá del género. Nuevos enfoques de "nuevas" dimensiones de la violencia en la pareja. [s.l.] Universitat Autònoma de Barcelona, 2004. 
CANTERA, L. M. Violencia En La Pareja: Fenómenos, Procesos y Teorías. Maltrato de género, infantil y de ancianos. Salamanca: Publicaciones Universidad Pontificia, 2005, p.55-94.

CANTERA, L. M.; ALVARADO, P. E. Claves en la investigación y análisis de los resultados, 2010, p. 25.

CANTERA, L. M; PALLARÈS, S; SELVA, C. Violencia. In: CANTERA, L. M.; PALLARÈS, C.Selva (Orgs.); Del malestar al bienestar laboral. Primera ed., Barcelona-España: Amentia, 2013, p.135-155.

CARO BLANCO, C. Un amor a tu medida. Estereotipos y violencia en las relaciones amorosas. Mujeres jóvenes en el siglo XXI, n. 83, 2008, p. 17.

COLLELL, J.; ESCUDÉ, C. Una aproximació al fenomen del maltractament entre alumnes (Bullying). ICVE. Revista Estudis de la Violència, n. 1, 2007, p. 1-10.

CUBELLS, J.; CALSAMIGLIA, A. El repertorio del amor romántico y las condiciones de posibilidad para la violencia machista. Romantic love's repertoire and the conditions of possibility of violence against women. v. 14, n. 5, 2015, p. 1681-1694.

DÍAZ-AGUADO, M. J. Por qué se produce la violencia escolar y cómo prevenirla. Revista Iberoamericana de Educación, v. 37, n. 37, 2005, p. 17-47.

DÍAZ-AGUADO, M. J. La juventud universitaria ante la igualdad y la violencia de género. Madrid-España: [s.n.]. 2012.

EXPÓSITO MOLINA, C. ¿Qué es eso de la interseccionalidad? Aproximación al tratamiento de la diversidad desde la perspectiva de género en España. Investigaciones Feministas, v. 3, 2012, p. 203-222.

FACIO, A. El Derecho a la Igualdad entre hombres y mujeres. In: Interpretación delos principios de igualdad y no discriminación para los derechos humanos de las mujeres en los instrumentos del Sistema Interamericano. [s.I: s.n.], 2009, p. 65-78.

FERNÁNDEZ, A. La violencia de género en adoslescentes crece un 37,2\%. Disponível: $<$ http://cadenaser.com/emisora/2016/02/12/radio_sevilla/1455277409_676653. html>.

GARAIGORDOBIL, M.; MARTINEZ-VALDERREY, V.; ALIRI, J. Autoestima, empatía y conducta agresiva en adolescentes víctimas de bullying presencial. European Journal of investigation in health, psychology and education, v. 3, 2013, n. 1, p. 29-40.

GIMENO, J.; PÉREZ, A. Métodos cuantitativos aplicados. Chihuahua: Centro de Investigación y Docencia, 2009.

GONZÁLEZ-ÁLVAREZ, M. et al. Adolescentes que agreden a sus padres. Un análisis descriptivo de los menores agresores. Psicopatología Clínica Legal y Forense, v. 10, 2010, p.37-53. 
GUO, P. Bullying, depression, and suicidal behaviors in adolescents: Secondary analysis of youth risk behavior survey data. [s.l: s.n.], 2013.

HERNANDO GÓMEZ, Á. La prevención de la violencia de género en adolescentes. Una experiencia en el ámbito educativo. Apuntes de Psicología, v. 25, n. 3, 2007, p. 325-340. JOLY, L.; CONNOLLY, J. Dating Violence among high-risk young women: A systematic review using quantitative and qualitative methods. Behavioral Sciences, v. 6, n. 1, 2016, p. 7. LAGARDE, M. Claves feministas para la negociación en el amor. Puntos de ed. Managua: [s.n.]. 2001.

MARTÍNEZ, M. L. et al. Violencia filio-parental: principales caracteristicas, factores de riesgo y claves para la intervencion. Papeles del psicólogo, v. 36, n. 3, 2015.

MIGUEL, A. DE. Feminismo y juventud en las sociedades formalmente igualitarias. Revista de Estudios de Juventud, n. 83, p. 1-23, 2008.

NAVARRO, K. Conflito, violencia y no violencia. Revista de Derecho y Ciencias Sociales, v. 6, 2013, p. 95-107.

OLWEUS, D. Conductas de acoso y amenazas entre escolares. $2^{\text {a }}$ ed. Madrid: [s.n.], 2004.

OMS: ORGANIZACIÓN MUNDIAL DE LA SALUD. Violencia juvenil. Disponível em: <http://www.who.int/mediacentre/factsheets/fs356/es/>. Acesso em: 29/5/2017, 2016.

ORTEGA, D. La violencia filio-parental. ¿un subtipo de violencia de género? Una revisión bibliográfica de la figura de la víctima. RES, Revista de Educación Social Educación Social y Género, n. July, 2015, p. 45-63.

PEÑA COLLAZOS, W. La violencia simbólica Como reproducción Biopolítica del poder. Revista Latinoamericana de Bioética, v. 9, 2009, p. 62-75.

PEREIRA DA SILVA, J.; SANTANA BARRETO, N. Violência escolar: problematizando a relação entre o bullying e a homofobia. GEPIADDE, Ano 6, v. 12, n. 2005, p. 15, 2012.

PEREIRA, R. et al. Propuesta de definición de violencia filio-parental: Consenso de la sociedad española para el estudio de la violencia filio-parental (SEVIFIP). Papeles del Psicólogo, v. 38, n. 3, 2017.

PUJAL LLOMBART, M.; GARCÍA-DAUDER, S. Desigualdades de género en "tiempos de igualdad". Aproximaciones desde dentro y fuera de la/s psicología/s. Quaderns de Psicologia, v. 12, n. 2, 2010, p. 7-20.

QUINTANA, A. Metodología de investigación científica cualitativa. In: QUINTANA PEÑA, A.; MONTGOMERY, W. (Eds.). Psicología: Tópicos de actualidad. Lima: [s.n.], 2006, p. 47-84. QUIÑONES, P.; CANTERA, L. M.; OJEDA, C. L. La violencia relacional en contextos laborales que trabajan contra la violencia. In: CANTERA, L. M.; PALLARÈS, S.; SELVA, C. (Eds.). Del malestar al bienestar laboral. Primera ed. Barcelona-España: Amentia, 2013. p. 135-155. 
RIGBY, K. Effects of peer victimization in schools and perceived social support on adolescent well-being. Journal of Adolescence, v. 23, n. 1, fev. 2000, p. 57-68.

RODRÍGUEZ, Y. et al. La fiabilidad y validez de la escala de mitos hacia el amor: las creencias de los y las adolescentes. Revista de Psicologia Social, v. 28, n. 2, 2013, p. 157-168.

RUÍZ OLABUÉNAGA, J. I. Metodología de la investigación cualitativa. Universida ed. Bilbao: [s.n.]. 2012.

RUIZ REPULLO, C. Voces tras los datos. Una mirada cualitativa a la violencia de género en adolescentes. Sevilla: [s.n.]. Disponível em: <www.juntadeandalucia.es/iam/>. 2016.

SANMARTÍN, J. ¿Qué es la violencia? Una aproximación al concepto y a la clasificación de la violencia. Daimon: Revista de filosofía, v. 2006, n. 42, 2007, p. 9-22.

SANTA CRUZ, I. Sobre el concepto de igualdad: algunas observaciones. Isegoría: Revista de filosofía moral y política, n. 6, 1992, p. 145-152.

SILVA SILVEIRA, R. DA; CAETANO NARDI, H. Interseccionalidade gênero, raça e etnia e a lei Maria da Penha. Psicologia \& Sociedade, v. 26, n. especial, 2014, p. 14-24.

SIMÓN, M. E. Hijas de la igualdad, herederas de injusticias. Madrid-España: Narcea, 2009.

STRAUSS, A.; CORBIN, J. Basics of qualitative research. Techniques and procedures for developing grounded theory. Editorial ed. Colombia: [s.n.], 2002.

SYMINGTON, A. Interseccionalidad: una herramienta para la justicia de género y la justicia económica. Derechos de las mujeres y cambio económico, v. 9, 2004, n. 8, p. 1-8.

UNIVERSITAT AUTÒNOMA DE BARCELONA. Codi de Bones Pràctiques en la recerca, 2013.

WILLIAMS CRENSHAW, K. Mapping the Margins: Intersectionality, Identity Politics, and violence against womwn of color. In: ALBERTSON FINEMAN, M.; MYKITIUK, R. (Eds.). The Public nature of private violence. London: Routledge, 1994. p. 93-118.

Marisa VÁzquez Martínez: Maestra, licenciada en psicología, Máster en terapia familiar sistémica, Posgrado en violencia familiar, Máster en investigación e intervención psicosocial y actualmente cursando el programa de doctorado en Persona y Sociedad en el mundo Contemporáneo de la Universidad Autónoma de Barcelona. Las áreas de interés son: educación, inclusión social, relaciones igualitarias, violencia de género. Co-autora de diversas publicaciones sobre estas temáticas.

E-mail: mariaisabel.vazquez@e-campus.uab.cat 
Alicia Pérez Tarrés: Graduada en Psicología por la Universidad de Barcelona, Máster en Psicología General Sanitaria y actualmente cursando el programa de doctorado en Persona y Sociedad en el mundo Contemporáneo de la Universidad Autónoma de Barcelona. Las áreas de interés son: sociedad, cultura, violencia, poder, género y autocuidado.

E-mail:ptt.alicia@gmail.com

Leonor M. Cantera Espinosa: Doctor of Philosophy (PhD) por la Universidad de Puerto Rico. Doctora en Psicología Social con Premio Extraordinario por la Universidad Autónoma de Barcelona. Profesora Titular de Psicología Social de la UAB y actual Directora del Departamento de Psicología Social. Coordinadora del grupo de investigación de la violencia en relaciones afectivas y en el trabajo-VIPAT. Sus áreas de interés son: violencia, género, trabajo, comunidad. Autora y co-autora de diversas publicaciones sobre estas temáticas.

E-mail: leonor.cantera@uab.es

Jollson Pereira da Silva: Doctor en Psicología por la Universidad Complutense de Madrid-España. Pos-Doctoral (Sênio) por la Universidad Autónoma de Barcelona-España. Profesor del Programa de Pos-Graduación en Psicología de la Universidad Federal de Sergipe (Brasil).

E-mail: joilsonp@hotmail.com 